\title{
Viabilidade econômica de vacas leiteiras F1 Holandês x Zebu com diferentes bases maternas e ordens de parto
}

\author{
[Economic viability F1 Holstein x Zebu cows of dairy from different maternal bases \\ and birth orders] \\ T. Martins Neto ${ }^{1}$, C.B. Silva ${ }^{1}$, J.R.M. Ruas ${ }^{2,4}$, E.A. Silva ${ }^{3}$, D.S. Queiroz ${ }^{3}$, \\ M.D. Costa $^{2}$, V.M. Gomes ${ }^{2}$, L.H.T. Santos ${ }^{1}$ \\ ${ }^{1}$ Aluno de pós-graduação - Universidade Estadual de Montes Claros - Montes Claros, MG \\ ${ }^{2}$ Universidade Estadual de Montes Claros - Janaúba, MG \\ ${ }^{3}$ Empresa de Pesquisa Agropecuária de Minas Gerais - Belo Horizonte, MG \\ ${ }^{4}$ Bolsista de Produtividade em Pesquisa CNPq
}

\begin{abstract}
RESUMO
Objetivou-se avaliar a viabilidade econômica de vacas F1 de Holandês x Zebu de diferentes bases maternas e ordens de parto. Foram utilizados dados do Campo Experimental da Epamig (FelixlândiaMG). Analisaram-se 406 lactações de vacas F1 H x Z de diferentes bases maternas. Estimaram-se a receita e os custos operacional efetivo, operacional total e custo total. As F1 Holandês x Gir tiveram médias de custos maiores ( $\mathrm{R} \$ 3582,30)$, seguidas pelas F1 Holandês x Nelogir ( $\mathrm{R} \$ 3448,06)$, e o menor foi das F1 Holandês x Nelore ( $\mathrm{R} \$ 3145,07)$. A receita total foi maior para as vacas F1 Holandês x Gir e F1 Holandês x Nelogir, com valores de R \$ 4394,96 e R \$ 4245,61, respectivamente, e a menor receita foi para F1 Holandês x Nelore, com valor de R\$ 3976,12. O lucro foi de R\$ 812, 65; R\$ 797,54 e R\$ 831,04 para as F1 filhas de base materna Gir, Nelogir e Nelore, respectivamente. Todas as bases maternas são viáveis economicamente e podem ser utilizadas no sistema de produção de leite e bezerros para venda. Todas as ordens de parto de Holandês x Zebu estudadas são viáveis economicamente.
\end{abstract}

Palavras-chave: gado mestiço, ordem de parto, rentabilidade, vacas leiteiras

\begin{abstract}
The objective was to evaluate the economic viability of a milk production system of F1 Holstein $x$ Zebu cows of different maternal bases and calving order. Lactations data of F1 Holtein $x$ Zebu cows $(n=406)$ of different maternal bases, from the experimental field of the EPAMIG at Felixlândia county (MG state, Brasil) were analyzed. Revenue, cost/effect of operation, total operating, and total cost have been estimated. F1 cows Holstein $x$ Gir had averages of higher costs $R \$ 3582.30$, followed by F1 cows Holstein $x$ Nelogir R\$ 3448.06; and the lowest was from cows F1 Holstein x Nellore with R\$ 3145.07. The total revenue was higher for Holstein $x$ Gir and Holstein $x$ Nelogir with recipe values of $R \$ 4394.96$ and $R \$ 4245.61$ respectively, and the lowest value of $R \$ 3976.12$ for Holstein $x$ Nellore. The profits were $R \$ 812.65 ; R \$ 797.54$ and $R \$ 831.04$ for animals of groups Holstein $x$ Gir, Holstein $x$ Nelogir and Holstein $x$ Nellore respectively. All genetic groups are economically viable and can be used in the production of milk and calves for sale. All calving order of Holstein $x$ Zebu studied are viable economically.
\end{abstract}

Keywords: mestizo cattle, birth orders, milk cows, profitability

\section{INTRODUÇÃO}

Cerca de $70 \%$ da produção de leite no Brasil provêm de vacas mestiças Holandês x Zebu. Entre os animais mestiços, as vacas F1 Holandês

Recebido em 19 de maio de 2017

Aceito em 3 de novembro de 2017

E-mail: teonetomartins@gmail.com x Zebu possuem produtividade média de 2.953 litros/vaca/ano, superior à produção média nacional, que é de 1.609 litros/vaca/ano (Vilela, 2011; Produção..., 2015). A escolha de vacas F1 Holandês x Zebu é uma opção que tem por vantagem o aumento da vida útil $\mathrm{e} a$ 
diversificação dos produtos finais, leite e bezerros de qualidade para venda. Com esses dois produtos, oscilações nos preços de leite podem ser compensadas pela venda de bezerros ou vice-versa (Ferreira et al., 2010). Porém, deve-se avaliar a eficiência técnica e econômica do rebanho leiteiro de acordo com a região em que se encontra.

A análise do custo de produção do leite desperta grande interesse, pois é decisiva na estratégia de negociação entre o produtor e o laticínio, bem como nas tomadas de decisões que envolvem o gerenciamento da fazenda, como nas decisões sobre a compra de rações; a compra, a venda e o descarte de animais; e o principal, que é detectar os pontos em que ocorrem perdas financeiras, eliminando-os, a fim de garantir que a atividade seja viável (Alves, 1999). Dessa forma, objetivou-se com este trabalho avaliar a viabilidade econômica de vacas F1 de Holandês $\mathrm{x}$ Zebu de diferentes bases maternas e ordem de parto, considerando suas características produtivas e reprodutivas.

\section{MATERIAL E MÉTODOS}

Foram utilizados dados da escrituração zootécnica do Campo Experimental de Felixlândia-MG (CEFX) pertencente à Epamig. O clima é classificado como tropical de savana, com duas estações bem distintas: inverno seco (maio a outubro) e verão chuvoso (novembro a abril). A precipitação pluviométrica média anual é de $1.126 \mathrm{~mm}$, e a temperatura média anual é de $22,9^{\circ} \mathrm{C}$ (Dados..., 2017). Os objetivos do sistema empregado no CEFX são a produção de leite e de bezerros para venda e a formação de pastos para alimentação do rebanho durante o ano. Os animais utilizados no CEFX para a produção leiteira são vacas $\mathrm{F} 1$ provenientes do cruzamento entre um touro da raça Holandesa com vacas zebuínas das raças Nelore e Gir Leiteiro e do seu cruzamento Nelore com Gir leiteiro (Nelogir). Quando as crias desse cruzamento - novilhas F1 - atingem o sexto mês de prenhez, passam pelo manejo de amansamento, facilitando o trabalho dos funcionários com as primíparas. A ordenha ocorre duas vezes ao dia, às sete e às 14 horas, e o sistema utiliza ordenha mecânica, com as vacas dispostas em fila indiana, separadas por fosso. No momento da ordenha, é fornecido concentrado para as vacas. Para facilitar a adaptação à ordenha, a cada 14 dias são feitas pesagens do leite produzido por vaca. O sistema utiliza bezerro ao pé como estímulo para a descida do leite, em que os bezerros com menos de dois meses mamam em uma teta enquanto as outras são ordenhadas. Após os dois meses de idade até o fim da lactação, os bezerros fazem apenas o apojo e mamam o leite residual. As vacas $\mathrm{F} 1$ são acasaladas com touros de comprovada fertilidade, que são colocados com elas logo após o parto, na proporção de um touro para cinquenta vacas. Os diagnósticos de gestação são realizados 45 dias após a cobrição. No sistema de produção de leite do CEFX, utilizam-se touros terminadores, animais das raças Nelore e Guzerá para as vacas multíparas, e, para a cobertura das novilhas, são utilizados touros da raça Gir. Todas as crias advindas desses cruzamentos são vendidas.

As vacas são mantidas em pastagens de Urochloa decumbens e Urochloa brizantha cv. Marandu durante o verão, distribuídas em piquetes em áreas de sequeiro ou irrigadas, recebendo suplementação mineral. Durante os meses mais secos e frios, as vacas recebem suplementação volumosa à base de silagem de milho. O concentrado com $22 \%$ de proteína bruta é fornecido somente para as vacas em lactação, no momento da ordenha e também junto com a silagem, e é ofertado de acordo com a produção leiteira. As vacas secas permanecem em pastagens divididas em vários piquetes, onde recebem apenas suplementação mineral.

Foram analisadas 406 lactações do período de janeiro de 2005 a dezembro de 2015, distribuídas entre as bases maternas e quatro ordens de parto (Tab. 1). Não foram consideradas as lactações encerradas antes dos seis meses, independentemente do motivo.

Os custos foram divididos em custo operacional efetivo (COE), custo operacional total (COT) e custo total (CT), segundo Gomes (2000). As receitas foram oriundas das vendas de leite e das crias. Para comparar os resultados obtidos sem que houvesse subestimação ou superestimação dos valores dos custos e das receitas nos diferentes anos, os valores de cada ano foram corrigidos para 01 de janeiro de 2016, conforme o índice geral de preços do mercado do Banco Central do Brasil (2017). 
Viabilidade econômica...

Tabela 1. Número de lactações de vacas F1 de acordo com a base materna e a ordem de parto

\begin{tabular}{ccccc}
\multirow{2}{*}{ Ordem de parto } & Gir & Nelogir & Nelore & Total \\
\cline { 2 - 5 } & 38 & 46 & 20 & 104 \\
$1^{\mathrm{a}}$ & 33 & 38 & 18 & 89 \\
$2^{\mathrm{a}}$ & 21 & 20 & 19 & 60 \\
$3^{\mathrm{a}}$ & 60 & 20 & 73 & 153 \\
$\geq^{\mathrm{a}}$ & 152 & 124 & 130 & 406 \\
\hline Total & &
\end{tabular}

Dos itens do COE e do COT, foram considerados comuns a todas as vacas os custos de mão de obra, reprodução, material de ordenha e higiene, energia, depreciação e reparo de máquinas e benfeitorias, controle sanitário e medicamentos, combustíveis e vestuários e utensílios de segurança dos funcionários. Impostos e taxas não entraram nos custos, pois a receita da venda do leite e de bezerros foi contabilizada com a dedução destes. $\mathrm{O}$ custo de mão de obra e o custo de reprodução em monta natural foram calculados de acordo com Costa et al. (2016).

Foram considerados custos individuais o COE e o COT, que variam, de uma vaca para outra, como custos de depreciação das vacas, custos do consumo de silagem, alimentação a pasto e custo de consumo de concentrado e mineral. $\mathrm{O}$ custo de depreciação das vacas foi calculado segundo a diferença entre o preço inicial e o preço final da vaca de descarte, dividido pelo tempo de permanência da vaca no rebanho; o valor inicial da vaca foi calculado de acordo com o preço da arroba de mercado da vaca em 01-01-2016 (Cepea, 2017), considerando-se o valor da vaca F1 Holandês x Nelore $(\mathrm{H} \times \mathrm{N})$ custando 1,3 vezes o preço da arroba de vaca de corte, a F1 Holandês x Nelogir ( $\mathrm{H}$ x NG) custando 1,6 vezes o preço da arroba de vaca de corte e a F1 Holandês x Gir ( $\mathrm{H}$ x G) custando 2 vezes o preço da arroba de vaca de corte. O consumo de silagem foi estimado conforme o peso de cada vaca (Nutrient..., 2001). Multiplicou-se o consumo pelo custo de produção da tonelada de silagem e pelo tempo de suplementação. O tempo de suplementação foi calculado considerando-se que foi metade dos dias que a vaca permaneceu em lactação mais 30 dias de período pré-parto. $\mathrm{O}$ custo do pasto foi estimado multiplicando-se o valor do litro de leite médio recebido no ano pelo tempo de permanência das vacas a pasto em dias. O tempo de permanência foi calculado como sendo o tempo de suplementação subtraído de 365 dias. O custo do consumo de concentrado foi estimado de acordo com a produção de leite das vacas, na relação de $1 \mathrm{~kg}$ de concentrado para $3 \mathrm{~kg}$ de leite produzido a partir de $5 \mathrm{~kg}$ de leite, multiplicado pelo preço de compra do concentrado com $22 \%$ de proteína. $\mathrm{O}$ custo do consumo de sal mineralizado foi estimado considerando-se um consumo de $20 \mathrm{~g}$ para cada $100 \mathrm{~kg}$ de peso vivo. Para o custo total (CT), foi incluído, além do COT, o custo de oportunidade de aplicação, que foi calculado conforme a taxa de juros fixada pelo Banco Central em 01-01-2016 (2017).

A receita oriunda da venda do leite foi estimada pelo cálculo da produção leiteira de cada vaca no ano multiplicado pelo preço médio pago pelo litro durante os anos analisados. Já a receita do bezerro foi estimada pelo cálculo do preço médio do bezerro vendido no ano, corrigido para o peso do bezerro aos 262 dias. As receitas da venda de leite e bezerros foram corrigidas para $o$ percentual de fertilidade das vacas de cada base materna, pois a quantidade de vacas do rebanho que estarão paridas e produzindo leite no próximo ano é dependente da fertilidade. Considerou-se que as vacas de primeira ordem de parto estavam com $100 \%$ de fertilidade, visto que a substituição nesse rebanho é feita com novilhas gestantes; já a fertilidade da segunda ordem de parto foi estimada considerando-se a fertilidade da primeira para a segunda ordem e, assim sucessivamente, para as demais ordens de parto. $\mathrm{O}$ percentual de fertilidade foi calculado considerando-se 365 dias dividido pelo número de dias de intervalo entre partos e multiplicado por 100 .

Foram avaliados os efeitos da base materna e da ordem de parto nos desempenhos produtivo, reprodutivo e econômico. As variáveis de desempenhos produtivo e reprodutivo foram o peso das vacas ao parto, o peso dos bezerros ao desmame, o percentual de fertilidade, a duração da lactação; os dados de desempenho econômico foram custos individuais, receitas e lucro, os quais foram submetidos à análise de variância 
(ANOVA), sendo utilizado o procedimento GLM do SAS (2003), e as médias foram comparadas pelo teste de Student-Newman Keuls a 5\% de probabilidade. As interações significativas foram desdobradas de acordo com os fatores envolvidos.

\section{RESULTADOS E DISCUSSÃO}

Não houve interação entre base materna e ordem de parto para peso das vacas ao parto, peso dos bezerros ao desmame, duração da lactação e produção total de leite, exceto para fertilidade (Tab. 2).
Quando se avaliou a ordem de parto, o peso das vacas foi menor $(\mathrm{P}<0,05)$ na primeira ordem, igual na segunda e na terceira, e superior na quarta ordem ou mais de parto. Entre as bases maternas, observou-se que as vacas $\mathrm{F} 1 \mathrm{H}$ x $\mathrm{G}$ tiveram menor $(\mathrm{P}<0,05)$ peso, enquanto as demais apresentaram médias superiores e similares entre si. Possivelmente tal resultado ocorreu pelo fato de a base materna Gir ser selecionada para leite e considerada de médio porte. Já as $\mathrm{F} 1 \quad \mathrm{H} \quad \mathrm{x} \quad \mathrm{NG}$ e $\mathrm{F} 1 \quad \mathrm{H} \quad \mathrm{x} \quad \mathrm{N}$ são descendentes de animais selecionados para corte; no caso, a F1 H x NG é neta de Nelore e a F1 H x $\mathrm{N}$ é filha de vaca Nelore de corte, que são bases maternas de maior porte (Raças..., 2015).

Tabela 2. Médias e coeficiente de variação (CV) das características de desempenhos produtivo e reprodutivo das vacas $\mathrm{F} 1$ de acordo com as bases maternas e a ordem de parto

\begin{tabular}{|c|c|c|c|c|c|c|}
\hline \multirow{2}{*}{$\begin{array}{c}\text { Bases } \\
\text { maternas }\end{array}$} & \multicolumn{3}{|c|}{ Ordem de parto } & \multirow[b]{2}{*}{$\geq 4^{\mathrm{a}}$} & \multirow[b]{2}{*}{ Média } & \multirow[b]{2}{*}{$\mathrm{CV}$} \\
\hline & $1^{\mathrm{a}}$ & $2^{a}$ & $3^{\mathrm{a}}$ & & & \\
\hline \multicolumn{7}{|c|}{ Peso das vacas ao parto $(\mathrm{kg})$} \\
\hline Gir & $480,2 \pm 45,6$ & $492,3 \pm 45,6$ & $500,8 \pm 47,9$ & $536,5 \pm 53,5$ & $502,5 \mathrm{~B}$ & \\
\hline Nelogir & $493,6 \pm 45,6$ & $533,1 \pm 53,7$ & $513,0 \pm 38,6$ & $564,6 \pm 71,6$ & $526,1 \mathrm{~A}$ & 10,1 \\
\hline Nelore & $495,8 \pm 56,0$ & $511,7 \pm 65,7$ & $537,8 \pm 55,5$ & $561,0 \pm 55,5$ & $526,6 \mathrm{~A}$ & \\
\hline Média & $489,9 c$ & $512,4 b$ & $517,2 \mathrm{~b}$ & $554,1 \mathrm{a}$ & & \\
\hline \multicolumn{7}{|c|}{ Peso dos bezerros ao desmame (kg) } \\
\hline Gir & $158,6 \pm 14,1$ & $163,0 \pm 14,1$ & $167,3 \pm 15,6$ & $169,8 \pm 22,3$ & $164,7 \mathrm{~A}$ & \\
\hline Nelogir & $159,3 \pm 12,8$ & $166,1 \pm 17,1$ & $164,1 \pm 18,2$ & $164,6 \pm 17,2$ & $163,5 \mathrm{~A}$ & 10,1 \\
\hline Nelore & $156,4 \pm 12,9$ & $173,2 \pm 18,3$ & $172,2 \pm 16,7$ & $168,9 \pm 15,6$ & $167,7 \mathrm{~A}$ & \\
\hline Média & $158,1 \mathrm{~b}$ & $167,4 \mathrm{a}$ & $167,9 \mathrm{a}$ & $167,8 \mathrm{a}$ & & \\
\hline \multicolumn{7}{|c|}{ Fertilidade (\%) } \\
\hline Gir & $100,0 \mathrm{~A} \pm 0$ & $88,5 \mathrm{~B} \pm 16,7$ & $95,3 \mathrm{~B} \pm 11,8$ & $101,7 \mathrm{~B} \pm 12,2$ & & \\
\hline Nelogir & $100,0 \mathrm{~A} \pm 0$ & $89,5 \mathrm{~B} \pm 14,0$ & $105,1 \mathrm{~A} \pm 8,5$ & $100,2 \mathrm{~B} \pm 10,4$ & & 10,6 \\
\hline Nelore & $100,0 \mathrm{~A} \pm 0$ & $101,9 \mathrm{~A} \pm 15,2$ & $110,2 \mathrm{~A} \pm 13,8$ & $112,1 \mathrm{~A} \pm 9,6$ & & \\
\hline Média & $100,0 \mathrm{a}$ & $93,3 \mathrm{~b}$ & $103,55 \mathrm{a}$ & $104,6 a$ & & \\
\hline \multicolumn{7}{|c|}{ Duração da lactação (dias) } \\
\hline Gir & $286,4 \pm 58,0$ & $295,2 \pm 42,4$ & $284,8 \pm 45,7$ & $296,8 \pm 34,9$ & $284,1 \mathrm{~A}$ & \\
\hline Nelogir & $287,4 \pm 48,9$ & $275,3 \pm 24,7$ & $275,0 \pm 31,1$ & $291,2 \pm 39,9$ & $282,2 \mathrm{~A}$ & 14,5 \\
\hline Nelore & $249,6 \pm 49,3$ & $249,6 \pm 26,2$ & $254,2 \pm 43,4$ & $244,8 \pm 24,9$ & $249,5 \mathrm{~B}$ & \\
\hline Média & $274,4 \mathrm{a}$ & $273,4 \mathrm{a}$ & $271,3 \mathrm{a}$ & $277,6 \mathrm{a}$ & & \\
\hline \multicolumn{7}{|c|}{ Produção total de leite (litros) } \\
\hline Gir & $2611,0 \pm 645,7$ & $3308,5 \pm 709,7$ & $3304,6 \pm 886,6$ & $3639,0 \pm 1037,1$ & $3216,0 \mathrm{~A}$ & \\
\hline Nelogir & $2515,7 \pm 687,1$ & $2938,4 \pm 610,7$ & $2778,7 \pm 516,5$ & $3317,5 \pm 589,7$ & $2887,6 \mathrm{~B}$ & 24,4 \\
\hline Nelore & $2037,6 \pm 620,7$ & $2345,5 \pm 499,0$ & $2462,8 \pm 601,1$ & $2963,3 \pm 600,8$ & $2452,3 \mathrm{C}$ & \\
\hline Média & $2388,4 \mathrm{c}$ & $2864,1 \mathrm{~b}$ & $2848,7 \mathrm{~b}$ & $3306,6 a$ & & \\
\hline
\end{tabular}

Médias, na linha, seguidas de letras minúsculas diferentes são diferentes $(\mathrm{P}<0,05)$ pelo teste de SNK.

Médias, na coluna, seguidas de letras maiúsculas diferentes são diferentes $(\mathrm{P}<0,05)$ pelo teste de $\mathrm{SNK}$.

Não houve diferença $(\mathrm{P}>0,05)$ entre os pesos dos bezerros à desmama para as diferentes bases maternas (Tab. 2), resultado diferente do encontrado por Mendes et al. (2010), os quais observaram que os bezerros filhos de vacas $\mathrm{F} 1 \mathrm{H}$ $\mathrm{x} \mathrm{N}$ foram mais pesados que os filhos de vacas F1 H x G. Para ordem de parto, apenas na primeira ordem de parto houve menor $(\mathrm{P}<0,05)$ peso dos bezerros. Uma possível explicação é que os bezerros filhos das vacas de primeira 
ordem são filhos de touro Gir; já nas demais ordens de parto, as vacas são acasaladas com touros terminadores da raça Nelore e Guzerá. Mendes et al. (2010) também observaram diferenças entre a primeira ordem de parto e as demais ordens, em que a ordem de parto influenciou no ganho de peso diário dos bezerros, com menor ganho observado nos bezerros das vacas de primeira ordem e, consequentemente, no peso final. Outro fato é que bezerros amamentam de leite residual e as vacas primíparas são de menores produções, o que pode ter contribuído para esse menor peso à desmama.

Entre as bases maternas, as vacas $\mathrm{F} 1 \mathrm{H} \times \mathrm{N}$ apresentaram maior $(\mathrm{P}<0,05)$ média de fertilidade na segunda e na quarta ordem ou mais de parto em relação às outras bases maternas. Porém, na terceira ordem de parto, as vacas F1 H x NG $(105,1 \%)$ e F1 H x N $(110,9 \%)$ tiveram médias de fertilidade superiores $(\mathrm{P}<0,05)$ às $\mathrm{F} 1$ $\mathrm{H} \times \mathrm{G}(95,3 \%)$. Entre as ordens de parto, a (\%) fertilidade da segunda ordem de parto foi menor $(\mathrm{P}<0,05)$ que as taxas fertilidade subsequentes. Segundo Borges et al. (2015), além de possuírem exigências nutricionais mais elevadas no pósparto, os animais de primeira para segunda ordem de parto encontram-se em crescimento e período de adaptação, tanto ao estado fisiológico de lactação quanto ao novo ambiente de manejo. Com isso, tendem a ter um período de serviço maior.

Quanto à duração da lactação, houve diferença $(\mathrm{P}<0,05)$ apenas entre as bases maternas (Tab. 2). Vacas $\mathrm{F} 1 \mathrm{H} \times \mathrm{N}$ tiveram menor média de duração da lactação; já as vacas F1 H x G e F1 H x NG tiveram duração de lactação semelhante entre si. Essa menor duração da lactação das F1 $\mathrm{H} \times \mathrm{N}$ se deve à maior fertilidade apresentada por elas $(110,9 \%$, Tab. 2), pois possuem um período de serviço menor. A duração da lactação para as vacas $\mathrm{F} 1 \mathrm{H}$ x $\mathrm{G}$ encontrada neste estudo foi menor que as encontradas na literatura, cerca de 302,2 dias (Balancin Júnior et al., 2014) e 306,5 dias (Gloria et al., 2006). Resultados semelhantes foram encontrados para animais F1 H x NG, cerca de 281,2 dias de duração da lactação (Raidan et al., 2015). Para F1 H x N, as médias encontradas no presente estudo foram levemente superiores às encontradas, 239,6 dias, por Glória et al. (2010).

Vacas F1 H x G apresentaram maior $(\mathrm{P}<0,05)$ média de produção total, seguidas pelas vacas $\mathrm{F} 1$ H x NG, cuja média, por sua vez, foi maior que a das $\mathrm{F} 1 \mathrm{H} \times \mathrm{N}$, demonstrando que houve influência das bases maternas, em que as médias de produção leiteira das filhas da base materna Gir, raça com seleção leiteira, foram superiores $(\mathrm{P}<0,05)$ às filhas de Nelore $\mathrm{e}$ às filhas de Nelogir, com produção intermediária às duas. Vale ressaltar que, mesmo nas vacas da base materna Nelore, que tiveram menor média de produção, a produção obtida ainda é maior que a média nacional de 1.609,00 litros/vaca/ano (Produção..., 2015). Em relação à ordem de parto, médias de produção total de leite da segunda e da terceira ordem de parto foram superiores $(\mathrm{P}<0,05)$ às médias da primeira ordem de parto e inferiores às médias da quarta ordem ou mais. Tal fato pode estar ligado à maturidade do animal, visto que os pesos das vacas na segunda e na terceira ordem de parto também foram semelhantes. Glória et al. (2010), ao estudarem a curva de lactação de vacas F1 de Holandês x Zebu em diferentes ordens de parto, observaram que a produção de leite é menor na primeira ordem e que houve aumento nas produções totais com o avançar das ordens de parto, corroborando os resultados encontrados neste estudo.

Para os custos com alimentação, houve interação $(\mathrm{P}<0,05)$ entre base materna e ordem de parto somente para o custo da silagem oferecida (Tab. 3).

Entre as bases maternas, as vacas $\mathrm{F} 1 \mathrm{H}$ x NG na quarta ordem ou mais de parto obtiveram custo médio da silagem oferecida maior $(\mathrm{P}<0,05)$ que nas demais ordens de parto (Tab. 3). Dentro de ordem de parto, vacas $\mathrm{F} 1 \mathrm{H}$ x $\mathrm{NG}$ na quarta ordem ou mais apresentaram custo superior $(\mathrm{P}<0,05)$ às demais bases maternas (Tab. 3). Vacas F1 H x NG possuem o peso médio maior $(\mathrm{P}<0,05)$ que o das vacas $\mathrm{F} 1 \mathrm{H} \times \mathrm{G}$ (Tab. 2) e, com isso, têm necessidade de mantença maior e duração da lactação maior $(\mathrm{P}<0,05)$ que as vacas F1 H x N (Tab. 2). Por essas razões, consomem maior quantidade de silagem no decorrer do ano. 
Tabela 3. Médias e coeficiente de variação (CV) das características de custo com alimentação das vacas F1 de acordo com as bases maternas e a ordem de parto

\begin{tabular}{|c|c|c|c|c|c|c|}
\hline \multirow{2}{*}{$\begin{array}{c}\text { Bases } \\
\text { materna } \\
\text { s }\end{array}$} & \multicolumn{4}{|c|}{ Ordem de parto } & \multirow[b]{2}{*}{ Média } & \multirow[b]{2}{*}{$\mathrm{CV}$} \\
\hline & $1^{\mathrm{a}}$ & $2^{\mathrm{a}}$ & $3^{a}$ & $\geq 4^{\mathrm{a}}$ & & \\
\hline \multicolumn{7}{|c|}{ Custo da silagem oferecida $(\mathrm{R} \$)$} \\
\hline Gir & $880,12 \mathrm{Aa} \pm 191,49$ & $900,98 \mathrm{Aa} \pm 181,28$ & $826,69 \mathrm{Aa} \pm 190,86$ & $876,42 \mathrm{~B} a \pm 145$, & & \\
\hline Nelogir & $917,33 \mathrm{Ab} \pm 170,5$ & $915,76 \mathrm{Ab} \pm 126,0$ & $908,26 \mathrm{Ab} \pm 176,5$ & $1085,52 \mathrm{Aa} \pm 207,3$ & & 19, \\
\hline Nelore & $848,52 \mathrm{Aa} \pm 177,04$ & $867,50 \mathrm{Aa} \pm 136,36$ & $804,81 \mathrm{Aa} \pm 203,24$ & $854,84 \mathrm{Ba} \pm 184,14$ & & \\
\hline \multicolumn{7}{|c|}{ Custo da alimentação em pasto (R\$) } \\
\hline Gir & $228.95 \pm 38.96$ & $218.44 \pm 33.16$ & $219.09 \pm 32.06$ & $235.11 \pm 29.00$ & 225.40B & \\
\hline Nelogir & $230.07 \pm 34.10$ & $231.50 \pm 22.23$ & $232.71 \pm 20.38$ & $226.32 \pm 24.76$ & 230.15B & 12. \\
\hline Nelore & $259.66 \pm 37.02$ & $251.36 \pm 24.47$ & $235.74 \pm 28.09$ & $252.28 \pm 27.69$ & 49.76 & \\
\hline Média & $239,56 \mathrm{a}$ & $233,76 \mathrm{a}$ & $229,18 \mathrm{a}$ & $237,90 \mathrm{a}$ & & \\
\hline \multicolumn{7}{|c|}{ Custo do consumo de concentrado $(\mathrm{R} \$)$} \\
\hline Gir & $471,94 \pm 219,42$ & $732,88 \pm 252,69$ & $752,24 \pm 277,11$ & $915,97 \pm 390,17$ & $\begin{array}{c}718,26 \\
\text { A }\end{array}$ & 40 , \\
\hline Nelogir & $431,47 \pm 230,09$ & $624,70 \pm 231,53$ & $561,48 \pm 172,85$ & $744,62 \pm 196,20$ & 590,57B & \\
\hline Nelore & $315,74 \pm 254,52$ & $438,97 \pm 195,54$ & $476,71 \pm 215,15$ & $695,68 \pm 225,57$ & $481,77 \mathrm{C}$ & \\
\hline Média & $406,38 \mathrm{c}$ & $598,85 \mathrm{~b}$ & $596,81 \mathrm{~b}$ & & & \\
\hline \multicolumn{7}{|c|}{ Custo do consumo de mineral (R\$) } \\
\hline Gir & $43,12 \pm 4,09$ & $44,20 \pm 4,05$ & $44,97 \pm 4,30$ & $48,17 \pm$ & $45,11 \mathrm{~B}$ & \\
\hline Nelogir & $32 \pm 4,09$ & $47,86 \pm 4,82$ & $46,06 \pm 3,47$ & $50,69 \pm$ & $47,23 \mathrm{~A}$ & 10, \\
\hline Nelore & $44,51 \pm 5,02$ & $45,95 \pm 5,90$ & $48,29 \pm 4,98$ & $50,37 \pm 4,98$ & $47,28 \mathrm{~A}$ & \\
\hline Média & $43,98 \mathrm{c}$ & $46,00 \mathrm{~b}$ & $46,44 \mathrm{~b}$ & $49,75 \mathrm{a}$ & & \\
\hline
\end{tabular}

Médias, na linha, seguidas de letras minúsculas diferentes são diferentes $(\mathrm{P}<0,05)$ pelo teste de $\mathrm{SNK}$.

Médias, na coluna, seguidas de letras maiúsculas diferentes são diferentes $(\mathrm{P}<0,05)$ pelo teste de SNK.

Vacas $\mathrm{F} 1 \mathrm{H}$ x $\mathrm{N}$ permaneceram mais tempo no pasto e, assim, tiveram um maior $(\mathrm{P}<0,05)$ custo de alimentação a pasto que as demais. $O$ custo de alimentação a pasto foi similar $(\mathrm{P}>0,05)$ entre as ordens de parto dentro de cada base materna. O custo da alimentação referente ao pasto teve influência da duração da lactação (Tab. 2), visto que vacas com maior duração da lactação permanecem mais tempo recebendo suplementação volumosa e, consequentemente, ficam menos tempo em pasto, inversamente às vacas com menor duração da lactação, que ficam mais tempo a pasto, pois, quando estão secas, não recebem suplementação volumosa.

O custo do consumo de concentrado é influenciado pela produção leiteira das vacas (Tab. 2) tanto na comparação de médias entre bases maternas quanto na comparação de médias entre as ordens de parto, pois as vacas mais produtoras recebem maiores quantidades de concentrado. No caso das bases maternas, as vacas $\mathrm{F} 1 \mathrm{H} \times \mathrm{G}$ tiveram o custo médio maior
$(\mathrm{P}<0,05)$, seguido pelas vacas $\mathrm{F} 1 \mathrm{H} \times \mathrm{NG}$ e, depois, pelas vacas F1 $\mathrm{H} \times \mathrm{N}$. Nas ordens de parto, o maior custo ficou com a quarta ordem ou mais de parto $(\mathrm{P}<0,05)$, seguido pela terceira e pela segunda ordem, que foram semelhantes, e pela primeira ordem, que obteve o menor custo. Segundo Costa et al. (2011), o fornecimento de concentrado de acordo com a produção leiteira é o recomendado a se fazer, pois, em quantidade fixa, pode subalimentar as vacas mais produtivas, diminuindo a produção de leite, e superalimentar as vacas menos produtivas, elevando os custos da alimentação.

Os custos com sal mineral apresentaram diferenças $(\mathrm{P}<0,05)$ entre as médias de base materna e ordem de parto e acompanharam o peso das vacas (Tab. 2), pois o consumo médio de sal mineral foi estimado pelo peso. As vacas F1 $\mathrm{H}$ x G obtiveram menor custo que as demais. $\mathrm{Na}$ ordem de parto, o custo de consumo de sal foi maior $(\mathrm{P}<0,05)$ na quarta ordem ou mais, 
intermediário na terceira e na segunda ordem, e menor na primeira.

$\mathrm{Na}$ determinação dos custos, foi obtido o valor médio do custo comum de $\mathrm{R} \$ 926,94$, para cada vaca (Tab. 4), correspondente ao somatório dos valores $\mathrm{R} \$ 652,46, \mathrm{R} \$ 63,26, \mathrm{R} \$ 33,12, \mathrm{R} \$$ 76,90, R\$35,13, R\$39,31, R \$21,33 e $\mathrm{R} \$ 5,43$, referentes a mão de obra, custo de reprodução, material de ordenha e higiene, custo de energia, reparo de máquinas e benfeitorias, controle sanitário e medicamentos, combustíveis e vestuários e utensílios de segurança dos funcionários, respectivamente. Este valor foi somado aos demais custos na determinação do custo operacional efetivo. Não houve interação entre base materna e ordem de parto para todas as características de custo das vacas (Tab. 4).

Tabela 4. Médias e coeficiente de variação (CV) dos custos das vacas F1 de acordo com as bases maternas e a ordem de parto

\begin{tabular}{|c|c|c|c|c|c|c|}
\hline \multirow{2}{*}{$\begin{array}{c}\text { Bases } \\
\text { maternas }\end{array}$} & \multicolumn{3}{|c|}{ Ordem de parto } & \multirow[b]{2}{*}{$\geq 4^{\mathrm{a}}$} & \multirow[b]{2}{*}{ Média } & \multirow[b]{2}{*}{$\mathrm{CV}$} \\
\hline & $1^{\mathrm{a}}$ & $2^{\mathrm{a}}$ & $3^{\mathrm{a}}$ & & & \\
\hline \multicolumn{7}{|c|}{1 - Custo operacional comum (Reais) } \\
\hline Todas & 926,94 & 926,94 & 926,94 & 926,94 & & \\
\hline \multicolumn{7}{|c|}{2 - Custo operacional individual (Reais) $=$ Custo total de alimentação } \\
\hline Gir & $1624.15 \pm 332.25$ & $1896.44 \pm 346.22$ & $1843.00 \pm 384.78$ & $2075.68 \pm 436.00$ & $1859.82 \mathrm{~A}$ & \\
\hline Nelogir & $1623.21 \pm 322.89$ & $1819.85 \pm 270.68$ & $1748.52 \pm 264.23$ & $2107.16 \pm 341.58$ & $1824.69 \mathrm{~A}$ & 18,7 \\
\hline Nelore & $1468.44 \pm 328.22$ & $1603.80 \pm 261.14$ & $1565.56 \pm 337.33$ & $1853.19 \pm 303.60$ & $1622.75 B$ & \\
\hline Média & $1571.93 \mathrm{c}$ & $1773.36 \mathrm{~b}$ & $1719.03 \mathrm{~b}$ & $2012.01 \mathrm{a}$ & & \\
\hline \multicolumn{7}{|c|}{$3-$ Custo operacional efetivo (Reais) $=$ Somatório de $1+2$} \\
\hline Gir & $2551.09 \pm 332.25$ & $2893,16 \pm 346.22$ & $2769.94 \pm 384.78$ & $3002.62 \pm 436.00$ & $2786.76 \mathrm{~A}$ & \\
\hline Nelogir & $2550.15 \pm 322.89$ & $2746.79 \pm 270.68$ & $2675.46 \pm 264.23$ & $3034.10 \pm 341.58$ & $2751.63 \mathrm{~A}$ & 12,4 \\
\hline Nelore & $2395.38 \pm 328.22$ & $2530.74 \pm 261.14$ & $2492.50 \pm 337.33$ & $2780.13 \pm 303.60$ & 2549.69B & \\
\hline Média & $2498.87 \mathrm{c}$ & $2700.30 \mathrm{~b}$ & $2645.97 \mathrm{~b}$ & $2938.95 \mathrm{a}$ & & \\
\hline \multicolumn{7}{|c|}{4 - Custo de depreciação de vacas (Reais) } \\
\hline Gir & $318,07 \pm 35,94$ & $326,05 \pm 29,92$ & $331,70 \pm 31,74$ & $355,52 \pm 35,46$ & $332,79 \mathrm{~A}$ & \\
\hline Nelogir & $228,62 \pm 21,12$ & $246,91 \pm 24,90$ & $237,59 \pm 17,92$ & $261,51 \pm 33,17$ & $243,66 \mathrm{~B}$ & 10,3 \\
\hline Nelore & $155,58 \pm 17,57$ & $160,59 \pm 20,63$ & $168,79 \pm 17,41$ & $176,06 \pm 17,42$ & $165,26 \mathrm{C}$ & \\
\hline \multicolumn{7}{|c|}{5 - Custo de depreciação de benfeitorias (Reais) } \\
\hline Todas & 105,48 & 105,48 & 105,48 & 105,48 & & \\
\hline \multicolumn{7}{|c|}{$6-$ Custo operacional total (Reais) $=$ Somatório de $3+4+5$} \\
\hline Gir & $2974.65 \pm 352.01$ & $3254.92 \pm 364.52$ & $3207.12 \pm 393.50$ & $3463.43 \pm 441.63$ & $3225.03 \mathrm{~A}$ & \\
\hline Nelogir & $2884.25 \pm 333.35$ & $3099.19 \pm 284.98$ & $3018.53 \pm 266.74$ & $3401.10 \pm 351.22$ & 3100.77B & 11,3 \\
\hline Nelore & $2656.44 \pm 342.80$ & $2796.81 \pm 277.91$ & $2766.78 \pm 350.31$ & $3061.68 \pm 310.64$ & $2820.43 \mathrm{C}$ & \\
\hline Média & $2838.45 \mathrm{c}$ & $3050.31 \mathrm{~b}$ & $2997.48 b$ & $3308.74 \mathrm{a}$ & & \\
\hline \multicolumn{7}{|c|}{7 - Custo de oportunidade da aplicação (Reais) } \\
\hline Gir & $252.84 \pm 29.92$ & $276.66 \pm 30.98$ & $272.60 \pm 33.44$ & $294.39 \pm 37.53$ & $274.12 \mathrm{~A}$ & \\
\hline Nelogir & $245.16 \pm 28.33$ & $263.43 \pm 24.22$ & $256.57 \pm 22.67$ & $289.09 \pm 29.85$ & $263.56 \mathrm{~B}$ & 11,3 \\
\hline Nelore & $225.79 \pm 29.13$ & $237.72 \pm 23.62$ & $235.17 \pm 29.77$ & $260.24 \pm 26.40$ & $239.73 \mathrm{C}$ & \\
\hline Média & $241.26 \mathrm{c}$ & $259.27 \mathrm{~b}$ & $254.78 \mathrm{~b}$ & $281.24 \mathrm{a}$ & & \\
\hline \multicolumn{7}{|c|}{$8-$ Custo total (Reais) $=6+7$} \\
\hline Gir & $3227.49 \pm 381.93$ & $3531.59 \pm 395.50$ & $3479.73 \pm 426.95$ & $3757.82 \pm 479.17$ & $3499.16 \mathrm{~A}$ & \\
\hline Nelogir & $3129.42 \pm 361.68$ & $3362.62 \pm 309.20$ & $3275.11 \pm 289.41$ & $3690.19 \pm 381.07$ & 3364.33B & 11,3 \\
\hline Nelore & $2882.24 \pm 371.94$ & $3034.54 \pm 301.54$ & $3001.96 \pm 380.08$ & $3321.92 \pm 337.04$ & $3060.16 \mathrm{C}$ & \\
\hline Média & $3079.72 \mathrm{c}$ & $3309.58 \mathrm{~b}$ & $3252.26 \mathrm{~b}$ & $3589.98 \mathrm{a}$ & & \\
\hline
\end{tabular}

Médias, na linha, seguidas de letras minúsculas diferentes são diferentes $(\mathrm{P}<0,05)$ pelo teste de SNK.

Médias, na coluna, seguidas de letras maiúsculas diferentes são diferentes $(\mathrm{P}<0,05)$ pelo teste de $\mathrm{SNK}$.

O custo total da alimentação apresentado na Tab. 4 é o resultado da soma de todos os custos médios com alimentação. Entre as bases maternas, as vacas $\mathrm{F} 1 \mathrm{H} \times \mathrm{N}$ obtiveram menor $(\mathrm{P}<0,05)$ custo total de alimentação; já as vacas F1 H x NG e F1 H x G foram semelhantes entre 
si. Nas ordens de parto, o custo total da alimentação da quarta ordem ou mais obteve médias maiores $(\mathrm{P}<0,05)$ que os demais; os custos da segunda e da terceira ordem de parto foram semelhantes entre si, e estas tiveram médias de custos superiores às médias da primeira ordem de parto.

Na depreciação das vacas, o valor é influenciado pelo valor de compra e descarte. Como as vacas F1 H x G têm maior valor de compra, sua depreciação anual também foi a maior entre as bases maternas, ficando intermediária para as vacas $\mathrm{F} 1 \mathrm{H}$ x $\mathrm{NG}$ e menor para as $\mathrm{F} 1 \mathrm{H}$ x N (Tab. 4). O custo de depreciação de benfeitorias foi calculado de forma coletiva e dividido pelo número de animais, o que gerou valor médio de $\mathrm{R} \$ 105,48$, considerado no cálculo individual. Segundo Reis et al. (2001), o cálculo da depreciação informa ao produtor o custo necessário para substituir os bens de capital quando estes se tornam inúteis em razão de desgaste físico ou econômico.

Para os valores médios obtidos no custo operacional total e no custo total (Tab. 4), vacas $\mathrm{F} 1 \mathrm{H} \times \mathrm{G}$ foram as que apresentaram maiores $(\mathrm{P}<0,05)$ custos, seguidas pelas $\mathrm{F} 1 \mathrm{H} \times \mathrm{NG}$, com custos intermediários, e pelas vacas $\mathrm{F} 1 \mathrm{H} \times \mathrm{N}$, com menores custos. Para ordem de parto (Tab. 4), tanto para custo operacional total quanto para custo total, a quarta ordem ou mais de parto teve custo maior $(\mathrm{P}<0,05)$ que as demais, seguida pelas terceira e segunda, que tiveram custos maiores que a primeira ordem de parto. Os custos mais elevados não representam necessariamente prejuízos ou menor lucro ao final de mês. Lopes et al. (2007) relatam que, na maioria das vezes, quando se aumenta a produção, aumenta-se também o custo total de produção, porém, se ocorrer melhor aproveitamento dos fatores produtivos e o aumento da produção for proporcionalmente maior que o aumento dos custos, isto indica uma taxa de crescimento da atividade.

O custo de mão de obra e os custos com alimentação suplementar representaram 18,64\%; $19,38 \% ; 21,32 \%$ e $53,15 \% ; 54,23 \%$ e $53,02 \%$ do custo total, para F1 H x G, F1 H x NG e F1 H x $\mathrm{N}$, respectivamente. Segundo Ferrazza et al. (2015), alimentação suplementar e mão de obra são itens que promovem efeitos representativos no custo total e operacional efetivo, corroborando os resultados encontrados neste estudo. Costa et al. (2016) observaram que os custos com maior participação foram mão de obra e alimentação suplementar dos animais $1 / 2$ Holandês x Zebu, que corresponderam a 23,34\% e $41,96 \%$ do custo total, respectivamente. O custo operacional efetivo, que são os gastos que implicam o desembolso direto do produtor para manutenção da atividade, representou 79,64\%; $81,78 \%$ e $83,31 \%$ do custo total para F1 H x G, F1 H x NG e F1 H x N, respectivamente, e, para as ordens de parto, representou $81,13 \% ; 81,59 \%$; $81,35 \%$ e $81,86 \%$ do custo total para primeira, segunda, terceira e quarta ou mais ordens de parto, respectivamente.

Para todos os itens de receita, não foram observadas interações $(\mathrm{P}>0,05)$ entre base materna e ordem de parto (Tab. 5).

A receita média oriunda da venda de leite por vaca foi maior $(\mathrm{P}<0,05)$ para as bases maternas F1 H x G e F1 H x NG (Tab. 5). O valor da receita de leite segue a produção total de leite (Tab. 2), e essa maior produção das vacas $\mathrm{F} 1 \mathrm{H} \mathrm{x}$ $\mathrm{G}$ e $\mathrm{F} 1 \mathrm{H} \times \mathrm{NG}$ se deve ao fato mencionado anteriormente de que estas são descendentes de vacas Gir, raça com seleção leiteira, e as F1 H x $\mathrm{N}$ são oriundas de vacas sem seleção para esta característica. Para as ordens de parto, os resultados também seguiram a produção total de leite por ordem de parto (Tab. 2), sendo a quarta ordem ou mais de parto a de maior $(\mathrm{P}<0,05)$ receita, seguida da terceira e segunda ordens, e a menor receita foi na primeira ordem de parto. Não foi observada interação entre base materna e ordem de parto para esta variável.

Entre as bases maternas, os valores obtidos com a venda de bezerros foram maiores $(\mathrm{P}<0,05)$ para as $\mathrm{F} 1 \mathrm{H} \times \mathrm{N}$ e $\mathrm{F} 1 \mathrm{H} \times \mathrm{NG}$. Como a receita proveniente da venda de bezerros está diretamente relacionada à taxa de fertilidade, visto que é contabilizada pelo número de animais em produção no rebanho, aquela que apresenta maior fertilidade, caso da $\mathrm{F} 1 \mathrm{H}$ x $\mathrm{N}$ e da $\mathrm{F} 1 \mathrm{H}$ x $\mathrm{NG}$ em algumas ordens de parto, resultou em maior número de crias, consequentemente maior receita. A ordem de parto também influenciou o valor de venda dos bezerros, uma vez que os valores obtidos para bezerros oriundos da primeira ordem foram menores $(\mathrm{P}<0,05)$ que nas demais ordens. Os menores valores na primeira ordem de parto está relacionado ao peso à 
desmama, que foi menor que nas demais. Para receita total, entre as bases maternas, a receita total média foi maior $(\mathrm{P}<0,05)$ para vacas $\mathrm{F} 1 \mathrm{H} \mathrm{x}$ G e F1 H x NG. Para as ordens de parto, a quarta ordem ou mais de parto teve maior receita $(\mathrm{P}<0,05)$, seguida pelas terceira e segunda ordens, e a menor receita foi das vacas de primeira ordem (Tab. 5).

Tabela 5. Médias e coeficiente de variação (CV) das características de receitas das vacas F1 de acordo com as bases maternas e ordem de parto

\begin{tabular}{|c|c|c|c|c|c|c|}
\hline \multirow{2}{*}{$\begin{array}{c}\text { Bases } \\
\text { maternas }\end{array}$} & \multicolumn{4}{|c|}{ Ordem de parto } & \multirow[b]{2}{*}{ Média } & \multirow[b]{2}{*}{$\mathrm{CV}$} \\
\hline & $1^{\mathrm{a}}$ & $2^{\mathrm{a}}$ & $3^{\mathrm{a}}$ & $\geq 4^{\mathrm{a}}$ & & \\
\hline \multicolumn{7}{|c|}{ Receita da venda de leite por vaca $(\mathrm{R} \$)$} \\
\hline Gir & $3119,00 \pm 789,59$ & $3351,05 \pm 842,82$ & $3539,39 \pm 929,67$ & $4349,19 \pm 1404,79$ & $3589,66 \mathrm{~A}$ & \\
\hline Nelogir & $3017,99 \pm 826,66$ & $3060,75 \pm 700,83$ & $3440,17 \pm 705,66$ & $3959,33 \pm 744,65$ & $3369,56 \mathrm{~A}$ & 10,9 \\
\hline Nelore & $2521,30 \pm 828,87$ & $2875,45 \pm 883,05$ & $3088,44 \pm 906,11$ & $3942,57 \pm 935,18$ & 3106,94B & \\
\hline Média & $2886,10 \mathrm{c}$ & $3095,75 \mathrm{bc}$ & $3356,00 \mathrm{~b}$ & $4083,70 \mathrm{a}$ & & \\
\hline \multicolumn{7}{|c|}{ Receita da venda de bezerros por vaca $(\mathrm{R} \$)$} \\
\hline Gir & $802,05 \pm 217,89$ & $825,91 \pm 250,08$ & $771,39 \pm 232.90$ & $821,85 \pm 225,34$ & $805,30 \mathrm{~B}$ & \multirow{4}{*}{24,0} \\
\hline Nelogir & $808,34 \pm 161,17$ & $865,06 \pm 182,37$ & $891,73 \pm 182,42$ & $939,06 \pm 284,92$ & $869,18 \mathrm{~A}$ & \\
\hline Nelore & $743,32 \pm 117,65$ & $917,90 \pm 218,14$ & $966,98 \pm 281,30$ & $848,50 \pm 161,94$ & $876,05 \mathrm{~A}$ & \\
\hline Média & $784,57 \mathrm{~b}$ & $968,62 \mathrm{a}$ & $876,70 \mathrm{a}$ & $869,80 \mathrm{a}$ & & \\
\hline \multicolumn{7}{|c|}{ Receita total por vaca $(\mathrm{R} \$)$} \\
\hline Gir & $3921,06 \pm 833,12$ & $4176,97 \pm 934,13$ & $4310,78 \pm 1036,76$ & $5171,04 \pm 1416,79$ & 4394,96A & \multirow{4}{*}{22,6} \\
\hline Nelogir & $3826,34 \pm 840,29$ & $3925,819 \pm 703,33$ & $4331,91 \pm 641,42$ & $4898,40 \pm 630,27$ & $4245,61 \mathrm{~A}$ & \\
\hline Nelore & $3264,63 \pm 800,17$ & $3793,36 \pm 921,10$ & $405543 \pm 1035,35$ & $4791,07 \pm 995,93$ & 3976,12B & \\
\hline Média & $3670,67 \mathrm{c}$ & $3965,38 b$ & $4232,71 \mathrm{~b}$ & $4953,50 \mathrm{a}$ & & \\
\hline
\end{tabular}

Médias, na linha, seguidas de letras minúsculas diferentes são diferentes $(\mathrm{P}<0,05)$ pelo teste de SNK.

Médias, na coluna, seguidas de letras maiúsculas diferentes são diferentes $(\mathrm{P}<0,05)$ pelo teste de $\mathrm{SNK}$.

A receita oriunda da venda de bezerros representou $18,32 \%$ da receita total para vacas F1 $\mathrm{H}$ x G, 20,47\% para vacas $\mathrm{F} 1 \mathrm{H} \times \mathrm{NG}$ e $22,03 \%$ para vacas F1 $\mathrm{H}$ x N. Moraes et al. (2004) relataram que a venda de bezerros de vacas F1 Holandês x Zebu representou cerca de $25 \%$ da receita total ao ano. Sem a receita da venda dos bezerros, o lucro seria de apenas $\mathrm{R} \$$ 90,49; R\$ 39,90 e R\$12,09 para as vacas F1 H x G, F1 H x N e F1 H x NG, respectivamente.

Para todos os itens da análise econômica, margem bruta, margem líquida, lucro, lucratividade e rentabilidade, não foram observadas interações $(\mathrm{P}>0,05)$ entre base materna e ordem de parto (Tab. 6).

Entre as bases maternas, os valores obtidos comportaram-se de forma similar $(\mathrm{P}>0,05) \mathrm{em}$ todos os itens avaliados (Tab. 6). Apesar de a receita total média da base materna Nelore ter sido a menor $(\mathrm{P}<0,05)$ das três (Tab. 5), seu custo médio também foi o menor $(\mathrm{P}<0,05)$ em comparação com as demais (Tab. 4). Isso contribuiu para que seus resultados de margem, lucro, lucratividade e rentabilidade fossem semelhantes $(\mathrm{P}>0,05)$ às demais bases maternas (Tab. 6), o que demonstra que nem sempre maior receita significa melhor desempenho econômico. Para ordens de parto, todos os itens avaliados apresentaram diferenças $(\mathrm{P}<0,05)$, sendo a quarta ordem ou mais de parto superior, seguida pela terceira ordem, que foi superior à segunda e à primeira ordem, isso para todas as variáveis da análise econômica. Apesar das diferenças, todos os resultados foram positivos (Tab. 6). 
Tabela 6. Médias e coeficiente de variação (CV) da margem bruta, margem líquida, lucro, lucratividade e rentabilidade obtidos por vaca $\mathrm{F} 1$ de acordo com a base materna e a ordem de parto

\begin{tabular}{|c|c|c|c|c|c|c|}
\hline \multirow{2}{*}{$\begin{array}{c}\text { Bases } \\
\text { maternas }\end{array}$} & \multicolumn{5}{|c|}{ Ordem de parto } & \multirow[b]{2}{*}{$\mathrm{CV}$} \\
\hline & $1^{\mathrm{a}}$ & $2^{\mathrm{a}}$ & $3^{\mathrm{a}}$ & $\geq 4^{\mathrm{a}}$ & Média & \\
\hline \multicolumn{7}{|c|}{ Margem bruta $(\mathrm{R} \$)$} \\
\hline Gir & $1369.97 \pm 551.00$ & $1353.58 \pm 871.27$ & $1540.83 \pm 784.86$ & $2168.41 \pm 1064.87$ & $1608,19 \mathrm{~A}$ & \\
\hline Nelogir & $1276.18 \pm 594.01$ & $1179.02 \pm 592.56$ & $1656.44 \pm 429.43$ & $1864.29 \pm 489.47$ & $1493,98 \mathrm{~A}$ & 47,0 \\
\hline Nelore & $869.25 \pm 544.74$ & $1262.62 \pm 783.47$ & $1562.92 \pm 759.75$ & $2010.94 \pm 795.21$ & $1426,43 \mathrm{~A}$ & \\
\hline Média & $1171.80 \mathrm{c}$ & $1265.07 \mathrm{c}$ & $1586.73 b$ & $2014.54 \mathrm{a}$ & & \\
\hline \multicolumn{7}{|c|}{ Margem líquida $(\mathrm{R} \$)$} \\
\hline Gir & $946.41 \pm 538.68$ & $922.04 \pm 879.89$ & $1103.65 \pm 784.46$ & $1707.60 \pm 1071.51$ & $1169,92 \mathrm{~A}$ & \\
\hline Nelogir & $942.08 \pm 596.73$ & $826.62 \pm 597.04$ & $1313.37 \pm 428.86$ & $1497.29 \pm 491.48$ & $1144,84 \mathrm{~A}$ & 60,9 \\
\hline Nelore & $608.18 \pm 536.37$ & $996.54 \pm 780.22$ & $1288.65 \pm 751.86$ & $1729.39 \pm 799.30$ & $1155,69 \mathrm{~A}$ & \\
\hline Média & $832.22 \mathrm{c}$ & $915.07 \mathrm{c}$ & $1235.22 b$ & $1644.76 \mathrm{a}$ & & \\
\hline \multicolumn{7}{|c|}{ Lucro (R\$) } \\
\hline Gir & $693.56 \pm 516.98$ & $645.38 \pm 882.09$ & $831.04 \pm 768.51$ & $1413.21 \pm 1045.51$ & $895,79 \mathrm{~A}$ & \\
\hline Nelogir & $696.91 \pm 580.16$ & $563.19 \pm 593.46$ & $1056.79 \pm 413.70$ & $1208.20 \pm 489.59$ & $881,27 \mathrm{~A}$ & 74,7 \\
\hline Nelore & $382.38 \pm 518.23$ & $758.81 \pm 771.68$ & $1053.47 \pm 730.44$ & $1469.15 \pm 785.99$ & $915,95 \mathrm{~A}$ & \\
\hline Média & $590.95 \mathrm{c}$ & $655.79 \mathrm{c}$ & $980.44 \mathrm{~b}$ & $1363.52 \mathrm{a}$ & & \\
\hline \multicolumn{7}{|c|}{ Lucratividade $(\%)$} \\
\hline Gir & $15.53 \pm 11.90$ & $12.29 \pm 18.30$ & $16.67 \pm 13.03$ & $23.74 \pm 16.94$ & $17,05 \mathrm{~A}$ & \\
\hline Nelogir & $15.82 \pm 13.10$ & $12.24 \pm 14.30$ & $23.73 \pm 5.57$ & $24.07 \pm 8.04$ & $18,96 \mathrm{~A}$ & 72,9 \\
\hline Nelore & $8.91 \pm 14.00$ & $16.83 \pm 17.15$ & $22.12 \pm 19.31$ & $28.54 \pm 12.15$ & $19,10 \mathrm{~A}$ & \\
\hline Média & $13.42 \mathrm{c}$ & $13.79 \mathrm{c}$ & $20.84 \mathrm{~b}$ & $25.45 \mathrm{a}$ & & \\
\hline \multicolumn{7}{|c|}{ Rentabilidade (\%) } \\
\hline Gir & $20.40 \pm 14.99$ & $18.71 \pm 23.92$ & $22.98 \pm 20.14$ & $36.02 \pm 23.56$ & $24,52 \mathrm{~A}$ & \\
\hline Nelogir & $21.37 \pm 17.22$ & $16.66 \pm 17.54$ & $31.82 \pm 10.32$ & $33.07 \pm 13.70$ & $25,73 \mathrm{~A}$ & 72,5 \\
\hline Nelore & $12.13 \pm 16.39$ & $24.50 \pm 22.68$ & $33.57 \pm 22.69$ & $43.47 \pm 21.54$ & $28,66 \mathrm{~A}$ & \\
\hline Média & $17.97 \mathrm{c}$ & $19.96 c$ & $29.46 b$ & $37.52 \mathrm{a}$ & & \\
\hline
\end{tabular}

Médias, na linha, seguidas de letras minúsculas diferentes são diferentes $(\mathrm{P}<0,05)$ pelo teste de SNK.

Médias, na coluna, seguidas de letras maiúsculas diferentes são diferentes $(\mathrm{P}<0,05)$ pelo teste de SNK.

\section{CONCLUSÃO}

Vacas F1 Holandês x Zebu de diferentes bases maternas são viáveis economicamente e podem ser utilizadas no sistema de produção de leite e bezerros. Ordem de parto é fator de interferência na rentabilidade, mas, em todas as ordens, verificou-se viabilidade econômica. Em rebanho F1 Holandês x Zebu, a receita oriunda da venda de bezerros é fundamental para o aumento do lucro.

\section{AGRADECIMENTOS}

À Epamig, pela concessão dos dados; à Capes, pela bolsa de mestrado; à Fapemig, pelo apoio financeiro (PPM 00558-16); ao Finep e MCTI, pelo apoio financeiro ao projeto $\mathrm{n}^{\circ} 1334 / 13$; ao INCT- Ciência Animal.

\section{REFERÊNCIAS}

ALVES, E. Leite: o que determinam os custos. Rev. Balde Branco, v.35, p.38-40, 1999

BALANCIN JÚNIOR, A.; PRATA, M.A.; MOREIRA, H.L. et al. Avaliação de desempenho produtivo e reprodutivo de animais mestiços do cruzamento Holandês x Gir. Bol. Ind. Anim., v.71, p.357-364, 2014.

BANCO CENTRAL DO BRASIL. Disponível em: <http://www.bcb.gov.br/pt-br/\#!/home>. Acessado em: 09 jan. 2017.

BORGES, Á.M.; MARTINS, T.M.; NUNES, P.P.; RUAS, J.R.M. Reprodução de vacas mestiças: potencialidade e desafios. Rev. Bras. Reprod. Anim., v.39, p.155-163, 2015. 
CEPEA - Centro de Estudos Avançados em Economia Aplicada. Disponível em: $<$ http://www.cepea.esalq.usp.br/br/indicador/boigordo.>. Acessado em: 09 jan. 2017.

COSTA, L.T.; SILVA, F.F.; VELOSO, C.M. et al. da. Análise econômica da adição de níveis crescentes de concentrado em dietas para vacas leiteiras mestiças alimentadas com cana-deaçúcar. Rev. Bras. Zootec., v.40, p.1155-1162, 2011.

COSTA, M.D.; RUAS, J.R.M.; MARTINS NETO, T. et al. Análise da relação benefíciocusto em sistema de produção de leite com animais mestiços no norte de Minas Gerais. Bol. Ind. Anim., v.73, p.244-251, 2016.

DADOS climáticos para cidades mundiais. Felixlândia: Climate-Data.Org. Disponível em $<$ https://pt.climate-data.org/location/176040/>. Acessado em: 18 jan. 2017.

FERRAZZA, R.A.; LOPES, M.A.; BRUHN, F.R.P.; MORAES, F. Índices de desempenho zootécnico e econômico de sistemas de produção de leite com diferentes níveis tecnológicos. Ciênc. Anim. Bras., v.16, p.193-204, 2015.

FERREIRA J.J.; RUAS, J.R.M.; CARVALHO, B.C. et al. Alimentação do rebanho F1: fator de menor custo na produção de leite. Vacas F1 Holandês x Zebu: produção eficiente de leite. Inf. Agropecu., v.31, n.258, 2010.

GLORIA，J.R.; BERGMAM，J.A.G.; REIS, R.B.; SILVA, M.A. Efeito da composição genética e de fatores de meio sobre a produção de leite, a duração da lactação e a produção de leite por dia de intervalo de partos de vacas mestiças Holandês-Gir. Arq. Bras. Med. Vet. Zootec. v.58, p.1139-1148, 2006.

GLÓRIA, J.R.; BERGMANN, J.A.G.; QUIRINO, C.R. et al. Curvas de lactação de quatro grupos genéticos de mestiças HolandêsZebu. Rev. Bras. Zootec., v.39, p.2160-2165, 2010 .
GOMES, S.T. Economia da produção do leite. Belo Horizonte: [s.n.], 2000.

LOPES, P.F.; REIS, R.P.; YAMAGUCHI, L.C.T. Custos e escala de produção na pecuária leiteira: estudo nos principais estados produtores do Brasil. Rev. Econ. Sociol. Rural., v.45, p.567590, 2007.

MENDES, G.A.; RUAS, J.R.M. et al. Potencial do rebanho leiteiro para a produção de bovinos de corte. Inf. Agropecu., v.31, p.101-111, 2010.

MORAES, A.C.A.; COELHO, S.G.; RUAS, J.R.M. et al. Estudo técnico e econômico de um sistema de produção de leite com gado mestiço $\mathrm{F}$ 1 Holandês-Zebu. Arq. Bras. Med. Vet. Zootec., v.56, p.745-749, 2004.

NUTRIENT requirements of dairy cattle. 7.ed. Washington: National Academy Press, 2001.

PRODUÇÃO da pecuária municipal. Rio de Janeiro: IBGE, v.43, 2015, 49p.

RAÇAS zebuínas. 2015. Disponível em: <http://www.abcz.org.br/Home

/Conteudo/23985-Racas-Zebuinas $>$. Acessado em: 4 fev. 2017.

RAIDAN, F.S.S.; COSTA, M.D.; RUAS, J.R.M. et al. Desempenho produtivo e reprodutivo de fêmeas F1 Holandês x Nelogir. Rev. Bras. Saúde Prod. Anim., v.16, p.678-687, 2015.

REIS, R.P.; MEDEIROS, A.L.; MONTEIRO, L.A. Custos de produção da atividade leiteira na região Sul de Minas Gerais. Organ. Rurais Agroind., v.3, [9p.], 2001.

SAS user's guide, version 9.1. Cary: SAS Inst., 2003.

VILELA, D. Sistemas de produção de leite para diferentes regiões do Brasil. Embrapa, 2011. Disponível em: <http://www.cnpgl.embrapa.br/ sistemaproducao/>. Acessado em: 2 fev. 2017. 